\title{
Multi-Wavelength Imaging of Galaxies - using Kiso Schmidt CCD Photometry
}

\author{
Wim van Driel \\ Kiso Observatory, Institute of Astronomy, The University of Tokyo, \\ Mitake-mura, Kiso-gun, Nagano-ken 397-01, Japan
}

\begin{abstract}
Equipping the $105 \mathrm{~cm}$ Kiso Observatory Schmidt telescope with large $C C D$ cameras has initiated a new phase for galaxy photometry studies performed with the telescope. As part of multi-wavelength studies deep multi-colour CCD images were obtained of various types of individual galaxies, as well as pairs and groups of galaxies.
\end{abstract}

\section{Observations}

During the last year about 30 galaxies observed previously at other wavelengths (radio, far-infrared) were observed with a single-chip CCD camera (Yoshida, this Volume) at the prime focus of the $105 \mathrm{~cm}$ Kiso Observatory Schmidt telescope as part of multi-wavelength imaging studies of various types of galaxies, in collaboration with astronomers from a number of countries.
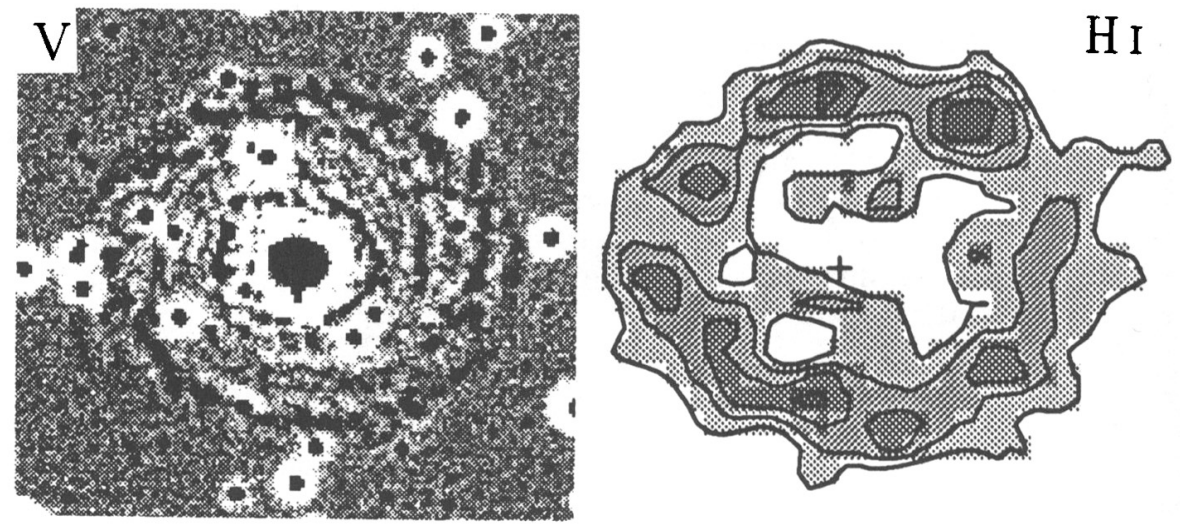

Figure 1. The axisymmetric ringed spiral galaxy NGC 7217 - (Left:) unsharp-masked V-band image showing the spiral and ring structures in the inner regions, and (right:) the $\mathrm{H}$ I column density distribution.

The combination of a large $(1000 \times 1018$ pixel $)$ CCD camera with a field-ofview of about 12.5 square and the fast, $\mathrm{f} / 3.1$ optics of one of the largest Schmidt telescopes in the world has provided us with a powerful instrument for obtaining deep, accurate surface photometry of galaxies of relatively large apparent diam- 
eter. The fast optics compensate the limited number of photometric nights, and the large field size allows the imaging of galaxies with large apparent diameters, for which the relatively poor seeing (typically $4^{\prime \prime}$ ) is less important. The Schmidt CCD camera has been proven to be especially suitable to reveal faint structures surrounding galaxies (e.g. Fig. 3).

\section{$\mathrm{HI}$ velocities}

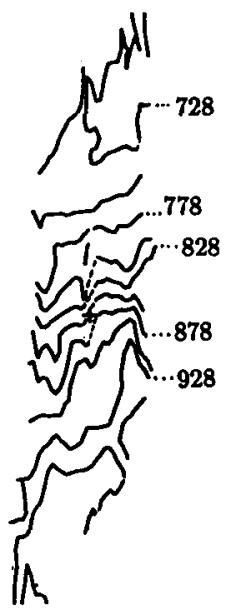

H I column density

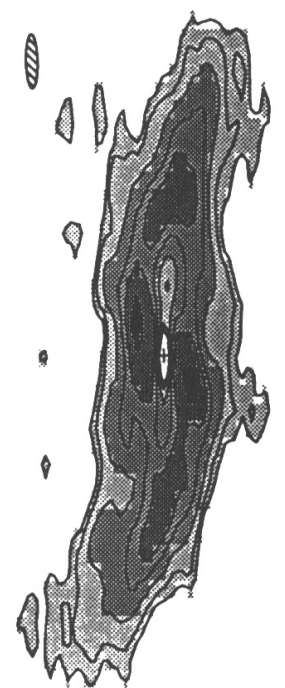

V

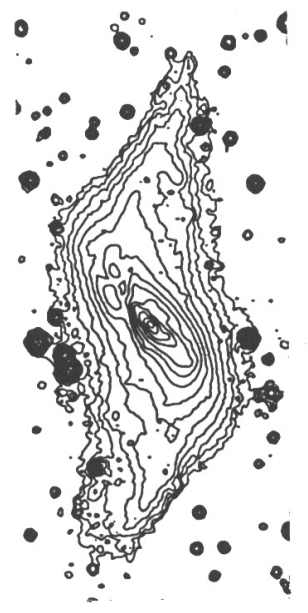

Figure 2. The nearby, unique polar ring galaxy NGC $660-\mathrm{H}$ I velocity field (left), H I column density distribution (middle), and deep V-band image, showing the inner disk (p.a. $45^{\circ}$ ) and outer polar ring. $\mathrm{H} \mathrm{I}, \mathrm{CO}$, and $\mathrm{H} \alpha$ line emission were detected in the inner spiral disk as well as in the polar ring.

The galaxies observed at Kiso were mapped previously at other wavelengths with a resolution of about $13^{\prime \prime}$ or more: in the $21 \mathrm{~cm} \mathrm{H} \mathrm{I} \mathrm{line} \mathrm{at} \mathrm{Westerbork,} \mathrm{in}$ the $\mathrm{CO}(1-0) /(2-1)$ lines at IRAM or Nobeyama, or at 50 and $100 \mu \mathrm{m}$ with the IRAS CPC detectors; TAURUS Fabry-Pérot $\mathrm{H} \alpha$ data or long-slit $\mathrm{H} \alpha$ spectra and near-infrared images were also obtained of some objects. Examples of the data obtained and of the types of galaxies studied are:

- Spiral galaxies with rings - for studies of the structure, origin, evolution, and dynamics of ring features in galactic disks. The example shown (Fig. 1) is NGC 7217, an isolated, symmetric Sab(r)-type spiral with 4 optical (pseudo-) rings (Buta et al. 1994). The $\mathrm{H} \mathrm{I}$ gas is concentrated in the strongest pseudoring, while CO line emission was detected in the innermost regions only. The formation mechanism of rings in such a symmetric object with flocculent spiral structure is not clear as it apparently lacks a non-axisymmetric potential.

Counter-rotation of the stars in NGC 7217 was reported by Kuijken (1993), who analysed the asymmetric stellar absorption lines in the inner regions, and interpreted them as counter-rotation of about $30 \%$ of the disk stars at any radius; 
a peculiar phenomenon, especially in such a nicely symmetric and undisturbed spiral. However, a crucial assumption here is that the bulge is rather faint and small, so all stars observed rotate in a flat disk. Our deep photometry shows, however, that the bulge is in fact (much) brighter than the disk at any radius. Thus, the bright and very extended bulge may cause the line profile asymmetry, while the stars in the disk all have the same sense of rotation.

- Polar ring galaxies and mergers - Polar ring galaxies are unique cases for studies of the three-dimensional shape of dark haloes around galaxies. Among these, NGC 660 (Fig. 2) is unique in itself, since it is by far the most nearby, allowing observations at relatively high resolution, and its inner (equatorial) disk is a gas-rich spiral, unlike the gas-poor lenticular-like disks found in practically all other polar ring galaxies. Thus, we can determine an accurate disk rotation curve from emission line data, rather than from less precise stellar absorption lines, which also need to be corrected for the asymmetric drift. Dynamical models were fitted to the morphological and kinematical data, in order to restrain the three-dimensional dark halo shape. This turned out to be impossible, however, since the disk and ring rotation curves do not have an overlapping region in radius; a common, but hitherto unrecognized, problem in polar ring galaxy studies. If we assume the halo to be spherical, its mass is about 0.6 times that of the luminous matter (van Driel et al. 1994a).

The nearby merger NGC 3310 (alias the 'bow-and-arrow' galaxy) is surrounded by a complex of faint arcs, though its large-scale $\mathrm{H}$ I kinematics are rather undisturbed (Mulder \& van Driel 1994), while the counter-rotating spiral NGC 4826 (the 'black eye' galaxy) shows a peculiar system of leading and trailing spiral arms (van Driel \& Buta 1993; Walterbos et al. 1994).

- Lenticulars (SO galaxies) - for studies of the origin and evolution of the gaseous component of these generally very gas-poor systems. The objects studied, which are among the most gas-poor galaxies mapped in the $21-\mathrm{cm} \mathrm{H} \mathrm{I} \mathrm{line,}$ do not appear to show the large outer $\mathrm{H}$ I rings found in the rare, truly gas-rich S0's, but rather centrally concentrated H I distributions. Near some of them gas-rich Irregular companions were detected.

- Pairs and groups - for studies of interactions and induced massive star formation activity in pairs and small groups of galaxies. One of the groups studied (NGC 7465) has an extreme amount of intergalactic H I gas, three times the mass within the galaxies themselves (van Driel et al. 1992). Our deep CCD images of the compact NGC $7463 / 64 / 65$ subgroup reveal faint arcs of luminous material around NGC 7465 , indicating that it is really a star-forming merger and not a quiescent elliptical, as it was (mis)classified earlier. The faint optical features correspond well with the H I structures detected by Li \& Seaquist (1994), see Fig. 3. The ring-like structure surrounding NGC 7465 may be a unique polarring, seen face-on (van Driel, $\mathrm{Li}$ et al. 1994, in preparation).

- Galactic disk opacity - a comparison of far-infrared, near-infrared, and optical scalelengths can, in principle, provide insight in the, controversial, issue of galactic disk opacity. For this purpose we use the IRAS CPC maps (van Driel et al. 1993) and published optical as well as Kiso CCD data. A clear interpretation of the data remains difficult at present, however, given the various, often contradictory, models available (van Driel et al. 1994b). 


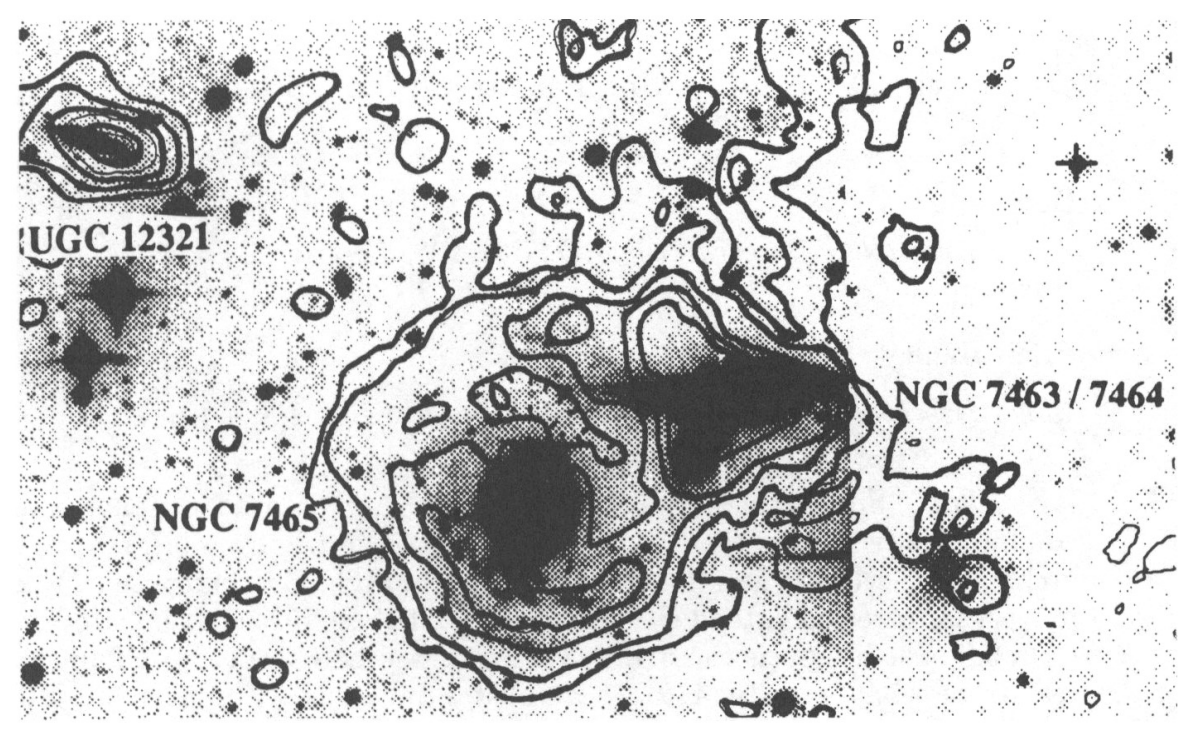

Figure 3. The interactive NGC $7463 / 64 / 65$ group - Overlay of the H I column density distribution (Li \& Seaquist 1994) on a deep Kiso Vband CCD image, and a digitized Palomar Sky Survey plate (the right, less dark, part of the frame). Our deep CCD image reveals a disturbed extension of the disk of NGC 7463, and a system of very faint, blue arcs around the previously misclassified elliptical NGC 7465 , which is in fact a starburst merger. These faint structures correspond well with the features in the superimposed $\mathrm{H}$ I column density contour map. The feature extending towards the NW of NGC $7463 / 64$ is an $\mathrm{H}$ I tail connected to nearby UGC 12313.

\section{References}

Buta R., van Driel W., Combes F., et al., 1994, ApJ, in press

Kuijken K., 1993, PASP, 105, 1016

Li J. G. \& Seaquist E. R., 1994, AJ, in press

Mulder P. S. \& van Driel W., 1994, A\&A, submitted

van Driel W., Augarde R., Bottinelli L., et al., 1992, A\&A, 259, 71

van Driel W. \& Buta R., 1993, PASJ, 45, L47

van Driel W., de Graauw Th., de Jong T. \& Wesselius P., 1993, A\&AS, 101, 207

van Driel W., Combes F., Casoli F., et al., 1994a, AJ, in press

van Driel W., Valentijn E. A., Kussendrager D. \& Wesselius P. R., 1994b, A\&AL, submitted

Walterbos R. A. M., Braun R. \& Kennicutt R. C., 1994, AJ, 107, 184 